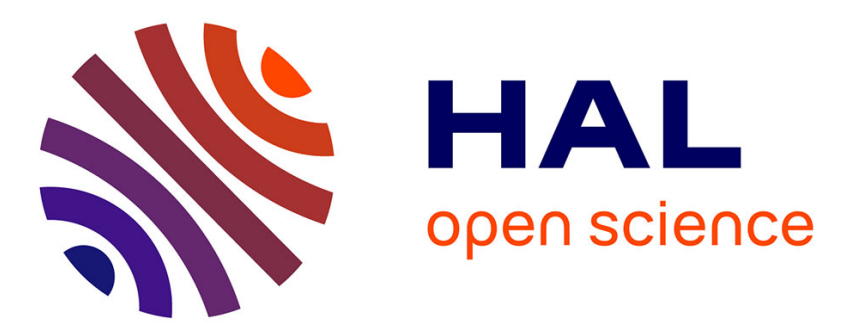

\title{
Computational Complexity of Rule Distributions of Non-uniform Cellular Automata
}

\author{
Alberto Dennunzio, Enrico Formenti, Julien Provillard
}

\section{To cite this version:}

Alberto Dennunzio, Enrico Formenti, Julien Provillard. Computational Complexity of Rule Distributions of Non-uniform Cellular Automata. Language and Automata Theory and Application, Mar 2012, Coruna, Spain. pp.204-215, 10.1007/978-3-642-28332-1_18 . hal-01297574

\section{HAL Id: hal-01297574 \\ https://hal.science/hal-01297574}

Submitted on 4 Apr 2016

HAL is a multi-disciplinary open access archive for the deposit and dissemination of scientific research documents, whether they are published or not. The documents may come from teaching and research institutions in France or abroad, or from public or private research centers.
L'archive ouverte pluridisciplinaire HAL, est destinée au dépôt et à la diffusion de documents scientifiques de niveau recherche, publiés ou non, émanant des établissements d'enseignement et de recherche français ou étrangers, des laboratoires publics ou privés. 


\title{
Computational Complexity of Rule Distributions of Non-Uniform Cellular Automata
}

\author{
Alberto Dennunzio ${ }^{2}$, Enrico Formenti ${ }^{1, \star}$, and Julien Provillard ${ }^{1}$ \\ 1 Université Nice-Sophia Antipolis, Laboratoire I3S, \\ 2000 Route des Colles, 06903 Sophia Antipolis (France). \\ \{enrico.formenti, julien.provillard\}@unice.fr \\ 2 Università degli Studi di Milano-Bicocca \\ Dipartimento di Informatica, Sistemistica e Comunicazione, \\ Viale Sarca 336, 20126 Milano (Italy) \\ dennunzio@disco.unimib.it
}

\begin{abstract}
CA are cellular automata which can have different local rules at each site of their lattice. Indeed, the spatial distribution of local rules completely characterizes $\nu$-CA. In this paper, sets of distributions sharing some interesting properties are associated with languages of biinfinite words. The complexity classes of these languages are investigated providing an initial rough classification of $\nu$-CA.
\end{abstract}

\section{Introduction}

Cellular automata (CA) are discrete dynamical systems consisting in an infinite number of finite automata arranged on a regular lattice. All automata of the lattice are identical and work synchronously. The new state of each automaton is computed by a local rule on the basis of its current state and the one of a fixed set of neighboring automata. This simple definition contrasts the huge number of different dynamical behaviors that made the model widely used in many scientific disciplines for simulating phenomena characterized by the emergency of complex behaviors from simple local interactions (particle reaction-diffusion, pseudo-random number generation, cryptography, etc.), see for instance [4].

In many cases, the uniformity of the local rule is more a constraint than a helping feature. Indeed, the uniformity constraint has been relaxed, for example, for modeling cell colonies growth, fast pseudo-random number generation, and VLSI circuit design and testing. This gave rise to new models, called non-uniform cellular automata $(\nu-\mathrm{CA})$ or hybrid cellular automata $(\mathrm{HCA})$, in which the local rule of the finite automaton at a given site depends on its position. If the study of dynamical behavior has just started up [36, applications and analysis of structural properties have already produced a wide literature (see [10[11]).

In this paper, we adopt a formal languages complexity point of view. Consider a finite set $\mathcal{R}$ of local rules defined over the same finite state set $A$. A (onedimensional) $\nu$-CA is essentially defined by the distribution or assignment of local

\footnotetext{
^ Corresponding author.
} 
rules in $\mathcal{R}$ to sites of the lattice. Whenever $\mathcal{R}$ contains a single rule, the standard cellular automata model is obtained. Therefore, each $\nu$-CA can be associated with a unique bi-infinite word over $\mathcal{R}$. Consider now the class $C$ of $\nu$-CA defined over $\mathcal{R}$ and sharing a certain property $P$ (for example surjectivity, injectivity, etc.). Clearly, $C$ can be identified as a set of bi-infinite words contained in ${ }^{\omega} \mathcal{R}^{\omega}$. In this paper, we analyze the language complexity of $C$ w.r.t. several well-known properties, namely number-conservation, surjectivity, injectivity, sensitivity to initial conditions and equicontinuity. We have proved that $C$ is a subshift of finite type and sofic, respectively, for the first two properties, while it is $\zeta$ rational for the last three properties in the list. Remark that for sensitivity to initial conditions and equicontinuity, the results are proved when $\mathcal{R}$ contains only linear local rules (i.e. local rules satisfying a certain additivity property) with radius 1 . The general case seems very complicated and it is still open.

In order to prove the main theorems, some auxiliary results, notions and constructions have been introduced (variants of De Bruijn graphs and their products, etc.). We believe that they can be interesting in their own to prove further properties.

In the paper, for lack of space most of proofs have been removed. They will appear in the long version of the paper. They can also be found at [5].

\section{Notations and Definitions}

For all $i, j \in \mathbb{Z}$ with $i \leq j$ (resp. $i<j$ ), let $[i, j]=\{i, i+1, \ldots, j\}$ (resp. $[i, j)=\{i, \ldots, j-1\})$.

Configurations and non-uniform automata. Let $A$ be a finite alphabet. A configuration or bi-infinite word is a function from $\mathbb{Z}$ to $A$. For any configuration $x$ and any integer $i, x_{i}$ denotes the element of $x$ at index $i$. The configuration set $A^{\mathbb{Z}}$ is usually equipped with the metric $d$ defined as follows

$$
\forall x, y \in A^{\mathbb{Z}}, d(x, y)=2^{-n} \text {, where } n=\min \left\{i \geq 0: x_{i} \neq y_{i} \text { or } x_{-i} \neq y_{-i}\right\} .
$$

The metric $d$ induces the Cantor topology on $A^{\mathbb{Z}}$. For any pair $i, j \in \mathbb{Z}$, with $i \leq j$, and any configuration $x \in A^{\mathbb{Z}}$ we denote by $x_{[i, j]}$ the word $w=x_{i} \ldots x_{j} \in$ $A^{j-i+1}$, i.e., the portion of $x$ inside $[i, j]$, and we say that the word $w$ appears in $x$. Similarly, $u_{[i, j]}=u_{i} \ldots u_{j}$ is the portion of a word $u \in A^{l}$ inside $[i, j]$ (here, $i, j \in[0, l))$. In both the previous notations, $[i, j]$ can be replaced by $[i, j)$ with the obvious meaning. For any word $u \in A^{*},|u|$ denotes its length. With $0 \in A$, a configuration $x$ is said to be finite if the number of positions $i$ at which $x_{i} \neq 0$ is finite.

A local rule of radius $r \in \mathbb{N}$ on the alphabet $A$ is a map from $A^{2 r+1}$ to $A$. Local rules are crucial in both the definitions of cellular automata and nonuniform cellular automata. A function $F: A^{\mathbb{Z}} \rightarrow A^{\mathbb{Z}}$ is a cellular automaton (CA) if there exist $r \in \mathbb{N}$ and a local rule $f$ of radius $r$ such that

$$
\forall x \in A^{\mathbb{Z}}, \forall i \in \mathbb{Z}, \quad F(x)_{i}=f\left(x_{[i-r, i+r]}\right) .
$$


The shift map $\sigma: A^{\mathbb{Z}} \rightarrow A^{\mathbb{Z}}$ defined as $\sigma(x)_{i}=x_{i+1}, \forall x \in A^{\mathbb{Z}}, \forall i \in \mathbb{Z}$ is one among the simplest examples of CA.

Let $\mathcal{R}$ be a set of local rules on $A$. A distribution on $\mathcal{R}$ is a function $\theta$ from $\mathbb{Z}$ to $\mathcal{R}$, i.e., a bi-infinite word on $\mathcal{R}$. Denote by $\Theta$ the set of all distributions on $\mathcal{R}$. A non-uniform cellular automaton $(\nu-\mathrm{CA})$ is a triple $\left(A, \theta,\left(r_{i}\right)_{i \in \mathbb{N}}\right)$ where $A$ is an alphabet, $\theta$ a distribution on the set of all possible local rules on $A$ and $r_{i}$ is the radius of $\theta_{i}$. A $\nu$-CA defines a global transition function $H_{\theta}: A^{\mathbb{Z}} \rightarrow A^{\mathbb{Z}}$ by

$$
\forall x \in A^{\mathbb{Z}}, \forall i \in \mathbb{Z}, \quad H_{\theta}(x)_{i}=\theta_{i}\left(x_{\left[i-r_{i}, i+r_{i}\right]}\right) .
$$

In the sequel, when no misunderstanding is possible, we will identify a $\nu$-CA with its global transition function. From [3], recall that a function $H: A^{\mathbb{Z}} \rightarrow A^{\mathbb{Z}}$ is the global transition function of a $\nu$-CA if and only if it is continuous. In this paper, we will consider distributions on a finite set of local rules. In that case, one can assume without loss of generality that there exists an integer $r$ such that all the rules in $\mathcal{R}$ have the same radius $r$. $\nu$-CA constructed on such finite sets of local rules are called $\mathrm{r} \nu$-CA (of radius $r$ ).

A finite distribution is a word $\psi \in \mathcal{R}^{n}$, i.e., a sequence of $n$ rules of $\mathcal{R}$. Each finite distribution $\psi$ defines a function $h_{\psi}: A^{n+2 r} \rightarrow A^{n}$ by

$$
\forall u \in A^{n+2 r}, \forall i \in[0, n), \quad h_{\psi}(u)_{i}=\psi_{i}\left(u_{[i, i+2 r]}\right) .
$$

These functions are called partial transition functions since they express the behavior of a $\nu$-CA on a finite set of sites: if $\theta$ is a distribution and $i \leq j$ are integers, then

$$
\forall x \in A^{\mathbb{Z}}, \quad H_{\theta}(x)_{[i, j]}=h_{\theta_{[i, j]}\left(x_{[i-r, j+r]}\right)} .
$$

Languages. Recall that a language is any set $\mathcal{L} \subseteq A^{*}$ and a finite state automaton is a tuple $\mathcal{A}=(Q, A, T, I, F)$, where $Q$ is a finite set of states, $A$ is the alphabet, $T \subseteq Q \times A \times Q$ is the set of transitions, and $I, F \subseteq Q$ are the sets of initial and final states, respectively. A path $p$ in $\mathcal{A}$ is a finite sequence $q_{0} \stackrel{a_{0}}{\longrightarrow} q_{1} \stackrel{a_{1}}{\longrightarrow} \ldots \stackrel{a_{n-1}}{\longrightarrow} q_{n}$ visiting the states $q_{0}, \ldots, q_{n}$ and with label $a_{0} \ldots a_{n-1}$ such that $\left(q_{i}, a_{i}, q_{i+1}\right) \in T$ for each $i \in[0, n)$. A path is successful if $q_{0} \in I$ and $q_{n} \in F$. The language $\mathcal{L}(\mathcal{A})$ of an automaton $\mathcal{A}$ is the set of the labels of all successful paths in $\mathcal{A}$. A language $\mathcal{L}$ is rational if there exists a finite automaton $\mathcal{A}$ such that $\mathcal{L}=\mathcal{L}(\mathcal{A})$.

A bi-infinite language is any subset of $A^{\mathbb{Z}}$. Let $\mathcal{A}=(Q, A, T, I, F)$ be a finite automaton. A bi-infinite path $p$ in $\mathcal{A}$ is a bi-infinite sequence $\ldots \stackrel{a_{-2}}{\longrightarrow} q_{-1} \stackrel{a_{-1}}{\longrightarrow}$ $q_{0} \stackrel{a_{0}}{\longrightarrow} q_{1} \stackrel{a_{1}}{\longrightarrow} \ldots$ such that $\left(q_{i}, a_{i}, q_{i+1}\right) \in T$ for each $i \in \mathbb{Z}$. The bi-infinite word $\ldots a_{-1} a_{0} a_{1} \ldots$ is the label of the bi-infinite path $p$. A bi-infinite path is successful if the sets $\left\{i \in \mathbb{N}: q_{-i} \in I\right\}$ and $\left\{i \in \mathbb{N}: q_{i} \in F\right\}$ are infinite. By similarity with the uniform case, we call this condition the Büchi acceptance condition. The bi-infinite language $\mathcal{L}^{\zeta}(\mathcal{A})$ of the automaton is the set of the labels of all successful bi-infinite paths in $\mathcal{A}$. A bi-infinite language $\mathcal{L}$ is $\zeta$-rational if there exists a finite automaton $\mathcal{A}$ such that $\mathcal{L}=\mathcal{L}^{\zeta}(\mathcal{A})$.

A bi-infinite language $X$ is a subshift if $X$ is (topologically) closed and $\sigma-$ invariant, i.e., $\sigma(X)=X$. For any $\mathcal{F} \subseteq A^{*}$ let $X_{\mathcal{F}}$ be the bi-infinite language 
of all bi-infinite words $x$ such that no word $u \in \mathcal{F}$ appears in $x$. A bi-infinite language $X$ is a subshift iff $X=X_{\mathcal{F}}$ for some $\mathcal{F} \subseteq A^{*}$. The set $\mathcal{F}$ is a set of forbidden words for $X$. A subshift $X$ is said to be a subshift of finite type (resp. sofic) iff $X=X_{\mathcal{F}}$ for some finite (resp. rational) $\mathcal{F}$.

For a more in deep introduction to the theory of formal languages, the reader can refer to [13] for rational languages, [16] for subshifts and 2122] for $\zeta$-rational languages.

Properties of non-uniform CA. A $\nu$-CA is sujective (resp. injective) iff its defining map $H: A^{\mathbb{Z}} \rightarrow A^{\mathbb{Z}}$ is surjective (resp. injective). A $\nu$-CA $H$ is equicontinuous if $\forall \varepsilon>0$ there exists $\delta>0$ such that for all $x, y \in A^{\mathbb{Z}}, d(y, x)<\delta$ implies that $\forall n \in \mathbb{N}, d\left(H^{n}(y), H^{n}(x)\right)<\varepsilon$. A $\nu$-CA $H$ is sensitive to the initial conditions (or simply sensitive) if there exists a constant $\varepsilon>0$ such that for any element $x \in A^{\mathbb{Z}}$ and any $\delta>0$ there is a point $y \in A^{\mathbb{Z}}$ such that $d(y, x)<\delta$ and $d\left(H^{n}(y), H^{n}(x)\right)>\varepsilon$ for some $n \in \mathbb{N}$.

Given a finite set of local rules $\mathcal{R}$, a predicate $P$ over distributions is a function from $\Theta$ to $\{\perp, \top\}$, where $\perp, T$ are the false and true symbols, respectively. In the sequel, we are interested in the complexity of the following language of bi-infinite words

$$
\mathcal{L}_{P}=\{\theta \in \Theta: P(\theta)=\top\} \text {. }
$$

\section{Number Conservation}

In physics, a lot of transformations are conservative: a certain quantity remains invariant during a whole experiment. Think to conservation laws of mass and energy for example. Both $C A$ and $\nu$-CA are used to represent phenomena from physics and it is therefore interesting to decide when they represent a conservative transformation. The case of uniform $\mathrm{CA}$ has been treated in [8, here we generalize those results to $\nu$-CA. Finally, we prove that the language of the set of distributions representing conservative $\mathrm{r} \nu$-CA is a subshift of finite type.

In this section, without loss of generality, $A$ is $\{0,1, \ldots, s-1\}$. Indeed, given any alphabet $A$, let $\phi: A \rightarrow \mathbb{N}$ be a morphism such that $0 \in \phi(A)$, then all the following results will hold by replacing $A$ by $\phi(A)$. Denote by $\underline{0}$ the configuration in which every element is 0 . For all configuration $x \in A^{\mathbb{Z}}$, define the partial charge of $x$ between the index $-n$ and $n$ as $\mu_{n}(x)=\sum_{i=-n}^{n} x_{i}$ and the global charge of $x$ as $\mu(x)=\lim _{n \rightarrow \infty} \mu_{n}(x)$. Clearly $\mu(x)=\infty$, if $x$ is not a finite configuration.

Definition 1 (FNC). $A \nu$ - $C A H$ is number-conserving on finite configurations $(F N C)$ if for all finite configurations $x, \mu(x)=\mu(H(x))$.

Remark that if $H$ is FNC then $H(\underline{0})=\underline{0}$ and, for any finite configuration $x$, $H(x)$ is a finite configuration.

Definition 2 (NC). A $\nu$-CA H is number-conserving (NC) if both the following conditions hold: 1) $H(\underline{0})=\underline{0}$, 2) $\forall x \in A^{\mathbb{Z}} \backslash\{\underline{0}\}, \lim _{n \rightarrow \infty} \frac{\mu_{n}(H(x))}{\mu_{n}(x)}=1$. 
Remark that if $x \neq \underline{0}$ the fraction $\frac{\mu_{n}(H(x))}{\mu_{n}(x)}$ is well-defined for $n$ sufficiently large.

In the general case, a $\nu$-CA can be FNC without being NC. Indeed, consider the following example.

Example 3. Let $H: A^{\mathbb{Z}} \rightarrow A^{\mathbb{Z}}$ be the $\nu$-CA such that for all configuration $x$, for all integer $i, H(x)_{2 i}=x_{i}$ and $H(x)_{2 i+1}=0$. Then $H$ is FNC but not NC. The configuration $\underline{1}$ in which every element is 1 verifies $\lim _{n \rightarrow \infty} \frac{\mu_{n}(H(\underline{1}))}{\mu_{n}(\underline{1})}=\frac{1}{2}$.

On the other hand, the following proposition shows that is not possible in the case of $\mathrm{r} \nu$-CA.

Proposition 4. Any $r \nu-C A$ of radius $r$ is $N C$ if and only if it is FNC.

Theorem 5. Let $\mathcal{R}$ be a finite set of local rules. Consider the predicate $P(\theta)=$ " $H_{\theta}$ is number conserving" over distributions $\theta \in \Theta$ on $\mathcal{R}$. Then, $\mathcal{L}_{P}$ is a subshift of finite type.

Proof. We are going to prove that $\mathcal{L}_{P}=X_{\mathcal{F}}$ where $\mathcal{F}$ is the following set $\left\{\psi \in \mathcal{R}^{2 r+1}: \exists u \in A^{2 r+1}, \psi_{2 r}(u) \neq u_{0}+\sum_{i=0}^{2 r-1} \psi_{i+1}\left(0^{2 r-i} u_{[1, i+1]}\right)-\psi_{i}\left(0^{2 r-i} u_{[0, i]}\right)\right\}$.

Assume that $\theta \in \mathcal{L}_{P}$ and let $j \in \mathbb{Z}$. For any $u \in A^{2 r+1}$, let $x, y$ be two finite configurations such that $x_{[j-r, j+r]}=u$ and $y_{[j-r, j+r]}=0 u_{[1,2 r]}$. As $H_{\theta}$ is NC, by Proposition 4, conditions $\mu(H(x))=\mu(x)$ and $\mu(H(y))=\mu(y)$ are true. So,

$$
\begin{gathered}
\sum_{i=0}^{2 r} \theta_{j+i-2 r}\left(0^{2 r-i} u_{[0, i]}\right)+\sum_{i=1}^{2 r} \theta_{j+i}\left(u_{[i, 2 r]} 0^{i}\right)=\sum_{i=0}^{2 r} u_{i}, \\
\sum_{i=1}^{2 r} \theta_{j+i-2 r}\left(0^{2 r-i+1} u_{[1, i]}\right)+\sum_{i=1}^{2 r} \theta_{j+i}\left(u_{[i, 2 r]} 0^{i}\right)=\sum_{i=1}^{2 r} u_{i} .
\end{gathered}
$$

Subtracting $\sqrt{2}$ from $\sqrt{1}$, we obtain $\theta_{j}(u)=u_{0}+\sum_{i=1}^{2 r} \theta_{j+i-2 r}\left(0^{2 r-i+1} u_{[1, i]}\right)-$ $\sum_{i=0}^{2 r-1} \theta_{j+i-2 r}\left(0^{2 r-i} u_{[0, i]}\right)$ which can be rewritten as

$$
\theta_{j}(u)=u_{0}+\sum_{i=0}^{2 r-1} \theta_{j+i+1-2 r}\left(0^{2 r-i} u_{[1, i+1]}\right)-\theta_{j+i-2 r}\left(0^{2 r-i} u_{[0, i]}\right) .
$$

Thus, for all $j \in \mathbb{Z}, \theta_{[j-2 r, j]} \notin \mathcal{F}$, meaning that $\theta \in X_{\mathcal{F}}$. So, $\mathcal{L}_{P} \subseteq X_{\mathcal{F}}$

Suppose now that $\theta \in X_{\mathcal{F}}$, i.e., for all integer $j, \theta_{[j-2 r, j]} \notin \mathcal{F}$. Taking $u=$ $0^{2 r+1}$, for all $j$ we have $\theta_{j+2 r}\left(0^{2 r+1}\right)=0+\sum_{i=0}^{2 r-1} \theta_{j+i+1}\left(0^{2 r+1}\right)-\theta_{j+i}\left(0^{2 r+1}\right)$ which leads to $\theta_{j}\left(0^{2 r+1}\right)=0$. For any finite configuration $x, \mu\left(H_{\theta}(x)\right)=$

$$
\begin{aligned}
& =\sum_{j \in \mathbb{Z}} H_{\theta}(x)_{j}=\sum_{j \in \mathbb{Z}} \theta_{j}\left(x_{[j-r, j+r]}\right) \\
& =\sum_{j \in \mathbb{Z}}\left(x_{j}+\sum_{i=0}^{2 r-1} \theta_{j+i+1-2 r}\left(0^{2 r-i} x_{[j-r+1, j-r+i+1]}\right)-\theta_{j+i-2 r}\left(0^{2 r-i} x_{[j-r, j-r+i]}\right)\right) \\
& =\sum_{j \in \mathbb{Z}} x_{j}+\sum_{i=0}^{2 r-1}\left(\sum_{j \in \mathbb{Z}} \theta_{j+i+1-2 r}\left(0^{2 r-i} x_{[j-r+1, j-r+i+1]}\right)\right. \\
& \left.\quad-\sum_{j \in \mathbb{Z}} \theta_{j+i-2 r}\left(0^{2 r-i} x_{[j-r, j-r+i]}\right)\right)
\end{aligned}
$$

Since $\sum_{j \in \mathbb{Z}} \theta_{j+i+1-2 r}\left(0^{2 r-i} x_{[j-r+1, j-r+i+1]}\right)=\sum_{j \in \mathbb{Z}} \theta_{j+i-2 r}\left(0^{2 r-i} x_{[j-r, j-r+i]}\right)$, we obtain $\mu\left(H_{\theta}(x)\right)=\sum_{j \in \mathbb{Z}} H_{\theta}(x)_{j}=\sum_{j \in \mathbb{Z}} x_{j}=\mu(x)$. Thus, $H_{\theta}$ is FNC and, by Proposition 4 NC. Hence, $\theta \in \mathcal{L}_{P}$. So, $X_{\mathcal{F}} \subseteq \mathcal{L}_{P}$. 


\section{Surjectivity and Injectivity}

In standard CA setting, injectivity is a fundamental property which is equivalent to reversibility [12. It is well-known that it is decidable for one-dimensional CA and undecidable in higher dimensions [114. Surjectivity is also a dimension sensitive property (i.e. decidable in dimension one and undecidable for higher dimensions) and it is a necessary condition for many types of chaotic behaviors.

In this paper, we prove that the language associated with distributions inducing surjective (resp. injective) $\nu$-CA is sofic (resp. $\zeta$-rational). Remark that constructions for surjectivity and injectivity are noticeably different, contrary to what happens for the classical CA when dealing with the decidability of those properties. Indeed in the uniform case, thanks to the Garden of Eden theorem [19]20, one construction is sufficient [23]. However the Garden of Eden theorem does not hold on $\nu$-CA [3], therefore distinct constructions are necessary.

Before proceeding to the main results of the section we need some technical lemma and new constructions. We believe that these constructions, inspired by [23], might be of interest in their own and could be of help for proving new results.

Lemma 6. For any fixed $\theta \in \Theta$, the $\nu-C A H_{\theta}$ is surjective if and only if $h_{\theta_{[i, j]}}$ is surjective for all integers $i, j$ with $i \leq j$.

Definition 7. Let $\mathcal{R}$ be a finite set of rules of radius $r$. The De Bruijn graph of $\mathcal{R}$ is the labeled multi-edge graph $\mathcal{G}=(V, E)$, where $V=A^{2 r}$ and edges in $E$ are all the pairs $(a w, w b)$ with label $(f, f(a w b))$, obtained varying $a, b \in A$, $w \in A^{2 r-1}$, and $f \in \mathcal{R}$.

Example 8. Let $A=\{0,1\}$ and consider the set $\mathcal{R}=\{\oplus, i d\}$ where $\oplus$ and $i d$ are the rules of radius 1 defined as $\forall x, y, z \in A, \oplus(x, y, z)=(x+z) \bmod 2$, and $i d(x, y, z)=y$. The De Bruijn graph of $\mathcal{R}$ is the graph $\mathcal{G}$ in Figure 1

Given two alphabets $A, B$ and a finite word $w=\left(a_{0}, b_{0}\right) \ldots\left(a_{n}, b_{n}\right) \in(A \times$ $B)^{*}$, the words $a=\operatorname{Pr}_{1}(w), b=\operatorname{Pr}_{2}(w)$, are the projections of $w$ on $A$ and $B$, respectively. By abuse of notation, we will write $(a, b) \in(A \times B)^{*}$ instead of $w \in(A \times B)^{*}, a=\operatorname{Pr}_{1}(w), b=\operatorname{Pr}_{2}(w)$. The same holds for bi-infinite words

Lemma 9. Let $\mathcal{G}$ be the De Bruijn graph of a finite set of rules $\mathcal{R}$. Consider $\mathcal{G}$ as an automaton where all states are both initial and final. Then, $\mathcal{L}(\mathcal{G})=$ $\left\{(\psi, u) \in(\mathcal{R} \times A)^{*}: h_{\psi}^{-1}(u) \neq \emptyset\right\}$.

Theorem 10. Let $\mathcal{R}$ be a finite set of local rules. Consider the predicate $P(\theta)=$ "H$H_{\theta}$ is surjective" over distributions $\theta \in \Theta$ on $\mathcal{R}$. Then $\mathcal{L}_{P}$ is a sofic subshift.

Proof. Set $\mathcal{F}=\left\{\psi \in \mathcal{R}^{*}: h_{\psi}\right.$ is not surjective $\}$. By Lemma 6, $\mathcal{L}_{P}$ is just the subshift $X_{\mathcal{F}}$. Consider the De Bruijn graph $\mathcal{G}$ of $\mathcal{R}$ as an automaton $\mathcal{A}$ where all states are both initial and final. By Lemma $9, \mathcal{L}(\mathcal{A})=\left\{(\psi, u) \in(\mathcal{R} \times A)^{*}\right.$ : $\left.h_{\psi}^{-1}(u) \neq \emptyset\right\}$. Build now the automaton $\mathcal{A}^{c}$ that recognizes $\mathcal{L}^{c}=\{(\psi, u) \in$ 


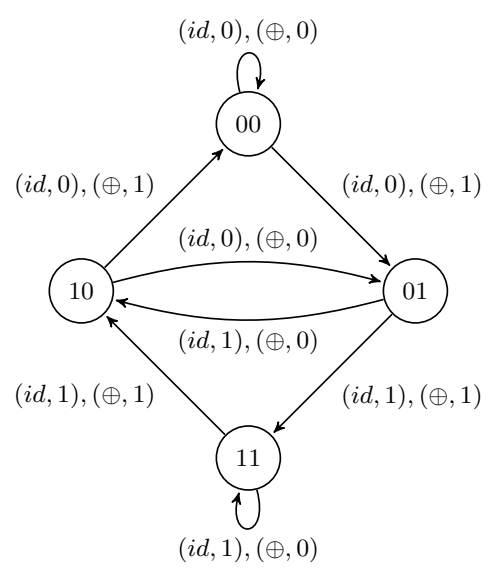

Fig. 1. De Bruijn graph of $\mathcal{R}=\{\oplus, i d\}$ (every printed edge represents two edges of the graph, labels are concatenated)

$\left.(\mathcal{R} \times A)^{*}: h_{\psi}^{-1}(u)=\emptyset\right\}$. Remove from $\mathcal{A}^{c}$ all second components of edge labels and let $\tilde{\mathcal{A}}$ be the obtained automaton. A word $\psi \in \mathcal{R}^{*}$ is recognized by $\tilde{\mathcal{A}}$ if and only if there exists $u \in A^{*}$ such that $(\psi, u) \in \mathcal{L}^{c}$, i.e., iff $h_{\psi}$ is not surjective. Thus, $\mathcal{L}(\tilde{\mathcal{A}})=\mathcal{F}$ and $\mathcal{L}_{P}=X_{\mathcal{F}}$ is a sofic subshift.

The proof of Theorem 10 provides an algorithm to build an automaton that recognizes the language $\mathcal{F}$ of the forbidden words for the sofic subshift $\mathcal{L}_{P}$ of distributions on a given finite set of rules $\mathcal{R}$. It consists of the following steps: 1 ) Build the De Bruijn graph $\mathcal{G}$ of $\mathcal{R} ; 2$ ) Consider $\mathcal{G}$ as an automaton whose all states are both initial and final and determinize it to obtain the automaton $\mathcal{A}$; 3) Complete $\mathcal{A}$ if necessary and make all final states non-final and vice versa to obtain $\left.\mathcal{A}^{c} ; 4\right)$ Delete all second components of edge labels of $\mathcal{A}^{c}$ to obtain $\tilde{\mathcal{A}}$.

Example 11. With the set $\mathcal{R}$ from the Example 8 as input, this algorithm gives the automaton in Figure 2. Thus, we deduce that $\mathcal{F}=\mathcal{R}^{*} i d \oplus(\oplus \oplus)^{*} i d \mathcal{R}^{*}$ and $\mathcal{L}_{P}$ is the well-known even subshift.

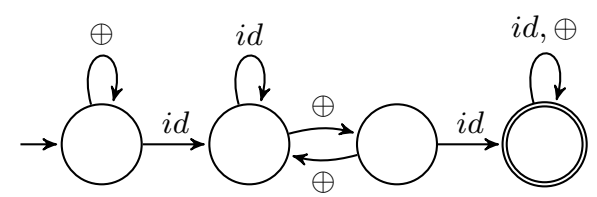

Fig. 2. The automaton $\tilde{\mathcal{A}}$ obtained from the set $\mathcal{R}=\{i d, \oplus\}$ 
In the final part of this section, we will use product graphs to study the injectivity property. Those graphs were first defined to deal with the decidability of the ambiguity of finite automata in [2].

Definition 12. Let $\mathcal{R}$ be a finite set of rules of radius $r$. Consider the De Bruijn graph $\mathcal{G}=(V, E)$ of $\mathcal{R}$. The product graph $\mathcal{P}$ of $\mathcal{R}$ is the labeled graph $(V \times V, W)$ where $\left(\left(u, u^{\prime}\right),\left(v, v^{\prime}\right)\right) \in W$ with label $(f, a) \in \mathcal{R} \times A$ if and only if $(u, v),\left(u^{\prime}, v^{\prime}\right) \in$ $E$ both with the same label $(f, a)$.

Remark 13. Any bi-infinite path in $\mathcal{P}$ with label $\left(\theta_{i}, z_{i}\right)_{i \in \mathbb{Z}} \in(\mathcal{R} \times A)^{\mathbb{Z}}$ corresponds to two bi-infinite paths in $\mathcal{G}$ in which the visited vertexes define two configurations $x$ and $y$ such that $H_{\theta}(x)=H_{\theta}(y)=z$.

We call quick-fail acceptance condition for bi-infinite paths in a finite automaton $\mathcal{A}$, the acceptance condition which accepts bi-infinite paths visiting at least once a final state. The set of labels of all such successful bi-infinite paths is said to be the language recognized by $\mathcal{A}$ under the quick-fail acceptance condition. We chose this terminology since the words belonging to the language recognized by $\mathcal{A}$ fail to induce injective $\nu$-CA (Theorem 15 .

Lemma 14. Let $\mathcal{A}=(Q, A, T, I, F)$ be a finite automaton. The bi-infinite language $\mathcal{L}$ recognized by $\mathcal{A}$ with the quick-fail acceptance condition is $\zeta$-rational.

Theorem 15. Let $\mathcal{R}$ be a finite set of local rules. Consider the predicate $P(\theta)=$ "H$H_{\theta}$ is injective" over distributions $\theta \in \Theta$ on $\mathcal{R}$. Then, $\mathcal{L}_{P}$ is $\zeta$-rational.

Proof. Let $\mathcal{P}$ be the product graph of $\mathcal{R}$. Consider now $\mathcal{P}$ as a finite automaton where all the states are initial and the final states are the pairs $\left(u, u^{\prime}\right)$ with $u \neq u^{\prime}$. Remove from $\mathcal{P}$ all second components of edge labels and let $\tilde{\mathcal{P}}$ be the obtained automaton. Then, the language recognized by $\tilde{\mathcal{P}}$ with the quick-fail acceptance condition is $\mathcal{L}_{P}^{c}$. By Lemma 14. $\mathcal{L}_{P}^{c}$ is $\zeta$-rational and, therefore, $\mathcal{L}_{P}$ is $\zeta$-rational too.

\section{$5 \quad$ Equicontinuity and Sensitivity for Linear $\mathrm{r} \nu$-CA}

Sensitivity to initial conditions is a widely known property indicating a possible chaotic behavior of a dynamical system and it is often popularized under the metaphor of butterfly effect [7]. At the opposite equicontinuity is an element of stability of a system. In this section, we are going to study these properties in the context of $\nu$-CA.

In the uniform case, equicontinuity points are characterized by blocking words 15. Some extensions have been made in the case of $\nu$-CA [3]. In general, the problem of establishing if a CA admits a blocking word is undecidable [9] but, in the case of linear CA, the problem turns out to be decidable [18. Moreover, the dichotomy theorem between sensitivity and presence of equicontinuity points [15] is not ensured in the context of $\nu$-CA. Therefore, in this preliminary 
study of the complexity of distributions, we preferred to focus on a sub-class in which the dichotomy theorem still holds, namely linear $\nu$-CA (Proposition 17).

In order to consider linear $\nu$-CA, the alphabet $A$ is endowed with a sum $(+)$ and a product $(\cdot)$ operation that make it a commutative ring and we denote by 0 and 1 the neutral elements of + and $\cdot$, respectively. Of course, $A^{n}$ and $A^{\mathbb{Z}}$ are also commutative rings where sum and product are defined component-wise and, with an abuse of notation, they will be denoted by the the same symbols.

Definition 16. A local rule $f$ of radius $r$ is said to be linear if and only if there exists a word $\lambda \in A^{2 r+1}$ such that $\forall u \in A^{2 r+1}, f(u)=\sum_{i=0}^{2 r} \lambda_{i} \cdot u_{i} . A \nu-C A H$ is said to be linear if it is defined by a distribution of linear local rules.

Proposition 17. Any linear $\nu-C A H$ is either sensitive or equicontinuous.

From now on we consider finite sets $\mathcal{R}$ in which all rules are linear of radius $r$.

Definition 18 (Wall). A right-wall is any element $\psi \in \mathcal{R}^{*}$ of length $n \geq r$ such that, for all word $v \in A^{r}$, the sequence $u_{\psi}(v): \mathbb{N} \rightarrow A^{n}$ recursively defined by $u_{\psi}(v)_{0}=0^{n}, u_{\psi}(v)_{1}=h_{\psi}\left(0^{r} u_{\psi}(v)_{0} v\right)$, and $u_{\psi}(v)_{k+1}=h_{\psi}\left(0^{r} u_{\psi}(v)_{k} 0^{r}\right)$ for $k>1$, verifies $\forall k \in \mathbb{N},\left(u_{\psi}(v)_{k}\right)_{[0, r-1]}=0^{r}$. Left-walls are defined similarly.

Roughly speaking, the sequence $u_{\psi}(v)$ gives the dynamical evolution of the function $h_{\psi}$ when the leftmost and rightmost inputs are fixed.

Lemma 19. For any right-wall $\psi \in \mathcal{R}^{n}$ and any $f \in \mathcal{R}$, both $f \psi$ and $\psi f$ are right-walls. Furthermore, if $\psi \in \mathcal{R}^{*}$ is a right-wall, then $\psi^{\prime} \psi \psi^{\prime \prime}$ is a right-wall for any $\psi^{\prime}, \psi^{\prime \prime} \in \mathcal{R}^{*}$. Similar results hold for left-walls.

Proposition 20. For any $\theta \in \Theta, H_{\theta}$ is sensitive if and only if one of the two following conditions holds: 1) There exists $n \in \mathbb{N}$ such that for all integer $m \geq n+r, \theta_{[n+1, m]}$ is not a right-wall; 2) There exists $n \in \mathbb{N}$ such that for all integer $m \leq-n-r, \theta_{[m,-n-1]}$ is not a left-wall.

The characterization of walls for the general case is still under investigation. However, remark that, given any set of linear local rules with radius $r \geq 1$ on a ring $(A,+, \cdot)$, it is possible to transform it in a set of linear local rules with radius 1 on a non-commutative ring. In this case, Lemma 19 and Proposition 20 remain true whereas the characterisation of walls of the Proposition 21 does not hold anymore. For these reasons, in the remaining part of this section, we will assume that $\mathcal{R}$ is a finite set of linear rules of radius 1 (over a commutative ring). In this case, any rule $f \in \mathcal{R}$ will be expressed in the following form: $\forall a, b, c \in A, f(a, b, c)=\lambda_{f}^{-} \cdot a+\tilde{\lambda}_{f} \cdot b+\lambda_{f}^{+} \cdot c$ for some $\lambda_{f}^{-}, \tilde{\lambda}_{f}, \lambda_{f}^{+} \in A$.

Proposition 21. A finite distribution $\psi \in \mathcal{R}^{n}$ is a right-wall (resp. a left-wall), if and only if $\prod_{i=0}^{n-1} \lambda_{\psi_{i}}^{+}=0$ (resp. $\left.\prod_{i=0}^{n-1} \lambda_{\psi_{i}}^{-}=0\right)$.

For any set $\mathcal{R}$ of linear rules of radius $r=1$, an automaton $\mathcal{A}=(Q, Z, T, I, F)$ recognizing walls can be constructed. The alphabet $Z$ is $\mathcal{R}$, the set of states $Q$ is $\{-,+\} \times A, I=\{(-, 0)\}, F=\{(+, 0)\}$ and the transition rule $T$ is as follows 
1. $\left((-, a), f,\left(-, \lambda_{f}^{-} \cdot a\right)\right), \forall a \in A \backslash\{0\}, \forall f \in \mathcal{R}$ (minimal left-wall detection).

2. $((-, 0), f,(-, 1)), \forall f \in \mathcal{R}$ (end of detection).

3. $((-, 1), f,(-, 1)), \forall f \in \mathcal{R}$ (waiting).

4. ((-, 1), f, (+, 1)), $\forall f \in \mathcal{R}$ (transition from left part to right part).

5. $((+, 1), f,(+, 1)), \forall f \in \mathcal{R}$ (waiting).

6. $((+, 1), f,(+, 0)), \forall f \in \mathcal{R}$ (beginning of detection).

7. $\left(\left(+, \lambda_{f}^{+} \cdot a\right), f,(+, a)\right), \forall a \in A \backslash\{0\}, \forall f \in \mathcal{R}$ (minimal right-wall detection).

Practically speaking, $\mathcal{A}$ consists of two components, the left and the right part, with a non-deterministic transition from left to right. Each component has two special states: the first one (the state $(-, 1)$ for the left part or $(+, 1)$ for the right part) on which $\mathcal{A}$ loops waiting for the detection of a minimal (wrt the length) wall, the second one on which $\mathcal{A}$ starts $((+, 0)$ for the right part) or ends $((-, 0)$ for the left part) the detection of such a wall. The graph structure of $\mathcal{A}$ is schematized in Figure 3 .

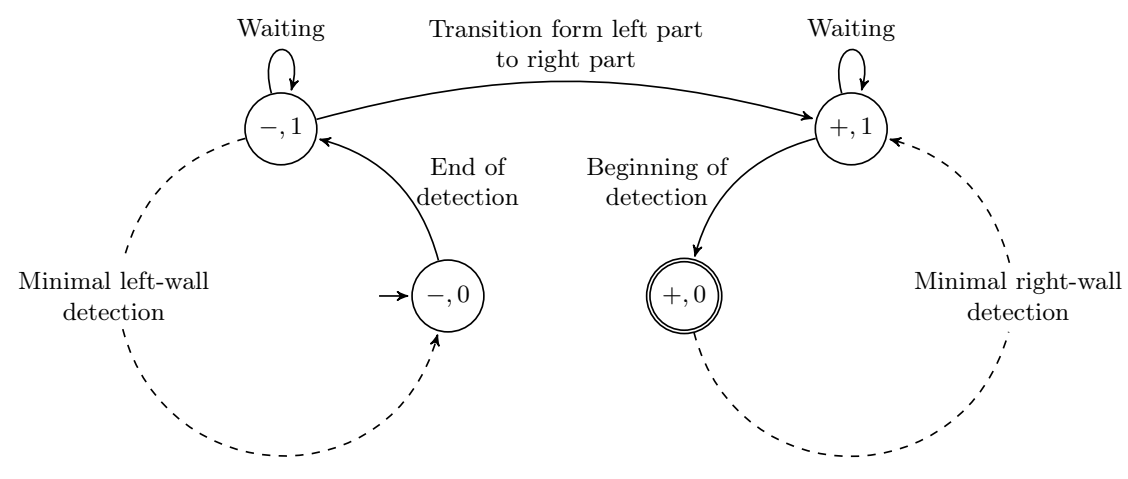

Fig. 3. Conceptual structure of the automaton $\mathcal{A}$ for walls detection

Theorem 22. Let $\mathcal{R}$ be a finite set of linear local rules of radius $r=1$. Consider the predicates $P_{1}(\theta)=$ "H $H_{\theta}$ is equicontinuous" and $P_{2}(\theta)=$ " $H_{\theta}$ is sensitive" over distributions $\theta \in \Theta$ on $\mathcal{R}$. Then, both $\mathcal{L}_{P_{1}}$ and $\mathcal{L}_{P_{2}}$ are $\zeta$-rational.

Proof. We are going to prove that $\mathcal{L}^{\zeta}(\mathcal{A})=\mathcal{L}_{P_{1}}$ where $\mathcal{A}$ is the automaton above introduced for the set $\mathcal{R}$ with Büchi acceptance condition. This permits to immediately state that $\mathcal{L}_{P_{1}}$ is $\zeta$-rational, and that, by Proposition $17, \mathcal{L}_{P_{2}}$ is $\zeta$-rational too.

Let $\theta \in \mathcal{L}^{\zeta}(\mathcal{A})$. We show that for any $n \in \mathbb{N}$, there exists $m \leq-n-1$ such that $\theta_{[m,-n-1]}$ is a left-wall. Let $n \in \mathbb{N}$. There is a successful path $p=\ldots \stackrel{\theta_{-1}}{\longrightarrow}$ $\left(s_{0}, a_{0}\right) \stackrel{\theta_{0}}{\longrightarrow}\left(s_{1}, a_{1}\right) \ldots$ in $\mathcal{A}$ and integers $i, j$ with $i<j<-n$ such that $\left(s_{i}, a_{i}\right)=$ $\left(s_{j}, a_{j}\right)=(-, 0)$ are two successive initial states. If $m \in(i, j)$ is the greatest 
integer with $\left(s_{m}, a_{m}\right)=(-, 1)$, the finite path $\left(s_{m}, a_{m}\right) \stackrel{\theta_{m}}{\longrightarrow}\left(s_{m+1}, a_{m+1}\right) \stackrel{\theta_{m+1}}{\longrightarrow}$ $\ldots \stackrel{\theta_{j-1}}{\longrightarrow}\left(s_{j}, a_{j}\right)$ is obtained by transitions of $\mathcal{A}$ from 1$)$. Then, $0=a_{j}=$ $a_{m} \cdot \prod_{l=m}^{j-1} \lambda_{\theta_{l}}^{-}$, and, by Proposition 21. $\theta_{[m, j-1]}$ is a left-wall. By Lemma 19 . $\theta_{[m,-n-1]}$ is a left-wall too. Similarly, it holds that for any $n \in \mathbb{N}$, there exists $m \geq n+1$ such that $\theta_{[n+1, m]}$ is a right-wall. Hence, by Propositions $20 H_{\theta}$ is equicontinuous, i.e., $\theta \in \mathcal{L}_{P_{1}}$.

Let $\theta \in \mathcal{L}_{P_{1}}$. By Proposition 20 , the sequence $\left(i_{k}\right)_{k \in \mathbb{Z}}$ such that $i_{0}=0$ and $\forall k \leq 0, i_{k-1}=\max \left\{j \in \mathbb{Z}: j<i_{k}\right.$ and $\theta_{\left[j, i_{k}-2\right]}$ is a left-wall $\}$, and $\forall k \geq$ $0, i_{k+1}=\min \left\{j \in \mathbb{Z}: j>i_{k}\right.$ and $\theta_{\left[i_{k}+2, j\right]}$ is a right-wall $\}$ is well-defined. For any $k<0, \theta_{\left[i_{k}, i_{k+1}-2\right]}$ is a left-wall and then $\prod_{j=i_{k}}^{i_{k+1}-2} \lambda_{\theta_{j}}^{-}=0$. So, for any $k<0$, setting $n=\min \left\{l \in \mathbb{Z}: \prod_{j=i_{k}}^{l} \lambda_{\theta_{j}}^{-}=0\right\}, p_{k}=(-, 1) \stackrel{\theta_{i_{k}}}{\longrightarrow}\left(-, \lambda_{\theta_{i_{k}}}^{-}\right) \stackrel{\theta_{i_{k}+1}}{\longrightarrow} \ldots \stackrel{\theta_{n}}{\longrightarrow}$ $\left(-, \prod_{j=i_{k}}^{n} \lambda_{\theta_{j}}^{-}\right) \stackrel{\theta_{n+1}}{\longrightarrow}(-, 1) \ldots \stackrel{\theta_{i_{k+1}-1}}{\longrightarrow}(-, 1)$ is a finite path in $\mathcal{A}$ from $(-, 1)$ to $(-, 1)$ with label $\theta_{\left[i_{k}, i_{k+1}-1\right]}$ which visits an initial state. Similarly, for any $k \geq 0$, there exists a finite path $p_{k}$ in $\mathcal{A}$ from $(+, 1)$ to $(+, 1)$ with label $\theta_{\left[i_{k}+1, i_{k+1}\right]}$ which visits a final state. Then, $p=\left(p_{k}\right)_{k \in \mathbb{N}}$ is a successful bi-infinite path in $\mathcal{A}$ with label $\theta$. Hence, $\theta \in \mathcal{L}^{\zeta}(\mathcal{A})$.

\section{Conclusions}

This paper investigates complexity classes associated to languages characterizing distributions of local rules in $\nu$-CA. Several interesting research directions should be explored.

First, we have proved that the language associated with distributions of equicontinuous or sensitive $\nu$-CA is $\zeta$-rational for the class of linear $\nu$-CA with radius 1 . It would be interesting to extend this result to sets of local rule distributions with higher radius. This seems quite difficult because this problem reduces to the study of the equicontinuity of $\nu$-CA of radius 1 on a non-commutative ring, loosing in this way "handy" results like Proposition 21.

Second, there is no complexity gap between sets of distributions which give injective $\nu$-CA and sensitive (plus the previously mentioned constraints). This is contrary to intuition since injectivity is a property of the global transition function whereas sensitivity is a property of its iterates. Indeed, we suspect that the characterization of distributions giving injective $\nu$-CA could be strengthened to deterministic $\zeta$-rational languages.

As a third research direction, it would be interesting to study which dynamical property of $\nu$-CA is associated with languages of complexity higher than $\zeta$-rational. We believe that sensitivity to initial conditions (with no further constraints) is a good candidate.

A further research direction would diverge from $\nu$-CA domain and investigate the topological structure of languages given by the quick-fail acceptance condition for finite automata in the vein of [17]. The authors have just started investigating this last subject. 


\section{References}

1. Amoroso, S., Patt, Y.N.: Decision procedures for surjectivity and injectivity of parallel maps for tessellation structures. J. Comput. Syst. Sci. 6(5), 448-464 (1972)

2. Berstel, J., Perrin, D.: Theory of Codes. Academic Press (1985)

3. Cattaneo, G., Dennunzio, A., Formenti, E., Provillard, J.: Non-uniform cellular automata. In: Language and Automata Theory and Applications, LATA 2009. Lecture Notes in Computer Science, vol. 5457, pp. 302-313 (2009)

4. Chaudhuri, P., Chowdhury, D., Nandi, S., Chattopadhyay, S.: Additive Cellular Automata Theory and Applications, vol. 1. IEEE Press (1997)

5. Dennunzio, A., Formenti, E., Provillard, J.: Local rule distributions, language complexity and non-uniform cellular automata. ArXiv e-prints (2011)

6. Dennunzio, A., Formenti, E., Provillard, J.: Non-uniform cellular automata: classes, dynamics, and decidability. ArXiv e-prints (2011)

7. Devaney, R.L.: An Introduction to Chaotic Dynamical Systems, 2nd Edition. Westview Pr (Short Disc) (2003)

8. Durand, B., Formenti, E., Róka, Z.: Number-conserving cellular automata i: decidability. Theoretical Computer Science 299(1-3), 523-535 (2003)

9. Durand, B., Formenti, E., Varouchas, G.: On undecidability of equicontinuity classification for cellular automata. DMTCS AB, 117-128 (2003)

10. Fúster-Sabater, A., Caballero-Gil, P., Pazo-Robles, M.: Application of linear hybrid cellular automata to stream ciphers. In: Computer Aided Systems Theory EUROCAST 2007. LNCS, vol. 4739, pp. 564-571. Springer (2007)

11. Gerlee, P., Anderson, A.R.A.: Stability analysis of a hybrid cellular automaton model of cell colony growth. Phys. Rev. E 75, 051911 (2007)

12. Hedlund, G.A.: Endomorphisms and automorphisms of the shift dynamical system. Theory of computing systems 3(4), 320-375 (1969)

13. Hopcroft, J.E., Motwani, R., Ullman, J.D.: Introduction to Automata Theory, Languages, and Computation (3rd Edition). Addison-Wesley (2006)

14. Kari, J.: Reversibility and surjectivity problems of cellular automata. Journal of Computer and System Sciences 48, 149-182 (1994)

15. Kůrka, P.: Languages, equicontinuity and attractors in cellular automata. Ergodic Theory and Dynamical Systems 17(2), 417-433 (1997)

16. Lind, D., Marcus, B.: An introduction to symbolic dynamics and coding. Cambridge University Press, New York, NY, USA (1995)

17. Litovsky, I., Staiger, L.: Finite acceptance of infinite words. Theoretical Computer Science 174, 1-21 (1997)

18. Manzini, G., Margara, L.: A complete and efficiently computable topological classification of d-dimensional linear cellular automata over $\mathrm{Zm}$. Theoretical computer science 221(1-2), 157-177 (1999)

19. Moore, E.F.: Machine models of self-reproduction. Proceedings of symposia in applied mathematics 14, 17-33 (1962)

20. Myhill, J.: The converse of moore's garden-of-eden theorem. Proceedings of the american mathematical society 14(4), 685-686 (1963)

21. Nivat, M., Perrin, D.: Ensembles reconnaissables de mots biinfinis. In: STOC. pp. 47-59. ACM (1982)

22. Perrin, D., Pin, J.E.: Infinite Words, Pure and Applied Mathematics, vol. 141. Elsevier (2004)

23. Sutner, K.: De Bruijn graphs and linear cellular automata. Complex Systems 5, 19-30 (1991) 